\title{
A Medicina Integrativa e a construção de um novo modelo na saúde
}

\author{
The Integrative Medicine \\ and the construction of a new health model
}

Márcia Aparecida Padovan Otani ${ }^{1}$

Nelson Filice de Barros ${ }^{2}$

${ }^{1}$ Faculdade de Medicina de Marília. Rua Luiz Padilha de Oliveira 285, Bairro Flândria. 17580-000 Pompeia SP. mm-otani@famema.br ${ }^{2}$ Universidade Estadual de Campinas.

\begin{abstract}
Integrative Medicine (IM) is the most recent concept on the Complementary and Alternative Medicines (CAM) debate, regarding the introduction and management of new practices in the health systems. This paper analyzed the different definitions of IM and its relation to CAM, through a systematic literature review on Medline, during the period of 1996 to 2005. The keyword "Integrative Medicine" was used and 36 papers were selected, which presented a definition of IM. It was identified that CAM is part of $I M$ and the definitions include: CAM and conventional medicine integration; use of evidence; combination of old healing systems and biomedicine; appreciation of the medical-patient relationship and communication; person as a whole conception; and focus on health, healing and prevention. It is concluded that the increasing interest of users, professionals and managers in CAM points out the need of developing the IM model, supporting the implementation and management of new care and healing practices.
\end{abstract}

Key words Integrative Medicine, Complementary and Alternative Medicine, Practices of health, Systematic review literature
Resumo Medicina Integrativa (MI) é o conceito mais recente no debate das Medicinas Alternativas e Complementares (MAC), em busca do modelo que viabilize introdução e gerenciamento de novas práticas nos sistemas nacionais de saúde. Este artigo analisou diferentes definições de MI e sua relação com as MAC, por meio de revisão sistemática da literatura no Medline, no período de 1996 a 2005. Foram utilizadas as palavras Integrative Medicine e selecionados 36 trabalhos que apresentaram definição de MI. Identificou-se que as MAC são parte da MI, e as definições abrangem: integração da medicina convencional com as MAC; utilização de evidências; combinação de antigos sistemas de cura com a biomedicina; valorização do relacionamento médico-paciente e da comunicação; consideração da pessoa por inteiro; e enfoque na saúde, na cura e na prevenção de doenças. Conclui-se que o crescente interesse de usuários, profissionais e gestores aponta a necessidade do desenvolvimento do modelo de MI, dando suporte à implementação e ao gerenciamento de novas práticas de cuidado e cura.

Palavras-chave Medicina Integrativa, Medicina Alternativa e Complementar, Práticas de saúde, Revisão sistemática da literatura 


\section{Introdução}

Os registros da história da medicina mostram que o cuidado em saúde teve diferentes modelos, desenvolvidos de acordo com o contexto e as bases culturais e materiais de cada época. O modelo ocidental atual é o biomédico, o qual apresentou fantásticas soluções para problemas da saúde e doença. No entanto, há algumas décadas tem sido fonte crescente de insatisfação da população, devido a sua dicotomia do cuidado e à superespecialização nas diversas áreas da medicina ${ }^{1}$.

O desencantamento com o modelo biomédico ou com a medicina convencional leva muitas pessoas a procurarem formas alternativas de tratamento, de modo que o número de profissionais que praticam outros modelos de cuidado e cura está em expansão.

O movimento de busca das práticas alternativas intensifica-se na década de 1960, motivado por vários outros fatores, como mudança do perfil de morbimortalidade, com a diminuição das doenças infectocontagiosas e aumento das doenças crônico-degenerativas em alguns países; aumento da expectativa de vida; crítica à relação assimétrica de poder entre médicos e pacientes, em que o profissional não fornece informações suficientes sobre o tratamento e cura do paciente; consciência de que a medicina convencional é deficiente para solucionar determinadas doenças, especialmente as crônicas; insatisfação com o funcionamento do sistema de saúde moderno, que inclui grandes listas de espera e restrições financeiras; informação sobre o perigo dos efeitos colaterais dos medicamentos e das intervenções cirúrgicas².

Esses fatores contribuíram para o desenvolvimento do modelo alternativo, ou seja, para a disseminação das ideias alternativas, relacionadas à lógica da alternância, assumindo ora um, ora outro aspecto. No campo da saúde, o modelo alternativo da medicina é compreendido como o polo oposto do modelo biomédico, pois enquanto a biomedicina investe para desenvolver a dimensão diagnóstica e aprofundar a explicação biológica, principalmente com dados quantitativos, a medicina alternativa volta-se para a dimensão da terapêutica, aprofundando-se nos problemas explicados pelas teorias do estilo de vida e ambiental ${ }^{3}$.

O acelerado crescimento das práticas alternativas trouxe tensões adicionais para o campo da saúde. Procurando harmonizar parte desses conflitos, no final dos anos 1980, nos Estados Unidos e no Reino Unido, foi adotada a denomi- nação Medicina Complementar, que significa "complemento", ou seja, "que sucede ao elementar" ção de modelos, fundada em conjunções aditivas ("e...e") em detrimento das conjunções alternativas ("ou...ou") ${ }^{3}$. Na década de 1990 foi criado, por exemplo, o National Center for Complementary and Alternative Medicine, nos Estados Unidos, que adota a seguinte definição para Medicina Alternativa e Complementar (MAC): Complementary and alternative medicine is a group of diverse medical and health care systems, pratices, and products that are not presently considered to be part of conventional medicine ${ }^{5}$.

No final da década de 1990, na tentativa de descrever um novo modelo de saúde que retrate a integração dos diversos modelos terapêuticos, mais do que simplesmente opere com a lógica complementar, e que ofereça o cuidado integral à saúde, foi criado o termo "Medicina Integrativa" (MI). A palavra "integração" significa o ato ou efeito de se integrar; ação ou política que visa integrar em um grupo as minorias raciais, religiosas, sociais ${ }^{6}$. Uma variedade de definições tem sido usada para descrever a ideia de integração entre as práticas convencionais e não convencionais, porém, atualmente, ainda se busca uma conceituação consistente.

O objetivo deste artigo é fomentar a discussão acerca do conceito de Medicina Integrativa, bem como sua relação com a Medicina Alternativa e Complementar, por meio da análise de publicações selecionadas em uma revisão sistemática da literatura no Medline/Pubmed.

\section{Metodologia}

A revisão sistemática da literatura é uma forma de síntese das informações disponíveis em dado momento, sobre um tema específico. Este método difere da revisão bibliográfica tradicional pelo fato de que na última o pesquisador revê os artigos que considera importantes, sem especificar ao leitor seus critérios de escolha. Portanto, a revisão sistemática busca superar os possíveis vieses em todas as etapas, seguindo um método rigoroso de busca e seleção das pesquisas, além de especificar, obrigatoriamente, os critérios de inclusão e exclusão dos artigos na pesquisa ${ }^{7}$.

Foi realizada uma pesquisa eletrônica da literatura na base de dados Medline, utilizando a palavra-chave "integrative medicine", para o período entre 01/01/1996 a 31/12/2005. Identificaram-se 236 trabalhos; porém, após a leitura dos 
títulos e resumos, foram selecionados 85, de acordo com os critérios de inclusão: artigos publicados no período; idiomas inglês, português e espanhol; tipos de estudo: ensaio clínico, carta, editorial, meta-análise, guia prático, ensaio controlado randomizado e revisão de literatura; apresentação da definição de Medicina Integrativa.

$\mathrm{O}$ acesso aos artigos deu-se por meio do Centro Latino-Americano e do Caribe de Informação em Ciências da Saúde (Bireme), Internet e contato direto com o autor. A primeira leitura dos artigos gerou um segundo corte, uma vez que foram identificadas 49 publicações que somente citaram genericamente a MI ou descreveram abordagem integrativa de práticas na clínica de determinada doença ou condição. Estão entre as perdas dois artigos não obtidos, mesmo após contato prévio com os autores.

$\mathrm{Na}$ segunda leitura foi realizada busca sistemática nas referências bibliográficas dos artigos lidos, com o propósito de localizar trabalhos não encontrados na pesquisa inicial. Assim, foram acrescentados outros cinco estudos, que preenchiam os critérios de inclusão, totalizando um número de 36 estudos para análise.

A partir da segunda leitura foram buscados os conceitos de MI apresentados pelos autores, bem como as relações que estabelecem com os conceitos de MAC. Outra volta ao material foi realizada e dela resultaram as classificações e os quadros apresentados a seguir.

\section{Resultados}

Os resultados foram divididos em duas partes. A primeira refere-se ao estudo das características gerais dos artigos selecionados por ano de publicação, local de realização dos trabalhos, formação do primeiro autor e tipo de produção (Quadro 1). Constatou-se nesta primeira análise: aumento significativo das produções nos últimos cinco anos; concentração da discussão nos EUA e predominância de médicos no debate. Em relação ao tipo de produção, classificado de acordo com a base de dados, observou-se a predominância do tipo "Revisão da literatura", que recebe este nome por abordar o tema referenciando-se a literatura. Em seguida vieram: o tipo "Editorial", que abre os volumes dos jornais apresentando questões que se colocam no debate atual; o tipo "Carta”, que tece comentários sobre artigos ou fatos recém-produzidos; o tipo "Ensaio clínico", que apresenta os resultados de investigação com metodologia específica; o tipo "Debate”, em que os autores discutem diferentes opiniões sobre o tema.

A segunda parte dos resultados refere-se à análise realizada no conteúdo de cada publicação, buscando a definição apresentada para Medicina Integrativa e a relação estabelecida com a MAC.

No conjunto dos artigos do tipo "Revisão de literatura", de acordo com o Quadro 2, constatase que, dos 22 trabalhos, somente um opõe-se à criação do termo Medicina Integrativa, pois, segundo Ernst ${ }^{33}$, os fundamentos apresentados por outros autores são uma duplicação de conceitos que estão no coração da medicina, sendo, portanto, necessário "curar" a medicina moderna de sua falta de holismo.

A maior parte dos artigos traz uma definição ampla de MI, ressaltando seus princípios fundamentados: na relação terapêutica ${ }^{20,30,36,38,39,43}$, na abordagem do paciente como um todo ${ }^{15,18,20-}$ $22,26,28,30,39,42,43$, na orientação para a cura $^{8,10,14,20,29,34,39,43}$ e na participação do paciente no tratamento ${ }^{8,30,31,38}$. Estes autores enfatizam a diferença de MI e MAC, reforçando que a perspectiva integrativa não significa a simples combinação da medicina convencional com as terapias complementares. Ao contrário, afirmam que a MI é a combinação da medicina convencional com a MAC, com base em evidências e com a finalidade de oferecer maior variedade de opções de tratamento aos pacientes ${ }^{10,14,23,24,28,38}$.

Outros temas foram relacionados com a MI. Dentre eles, destacam-se: o aumento no uso das MAC, quase sempre justificado pela insatisfação de pacientes e médicos com a abordagem biomédica $^{15,18,20,22-24,28,34,36}$; o debate sobre o ensino das MAC em escolas de medicina ${ }^{15,22,23,26,36,38,42}$; a necessidade de maior desenvolvimento e investimento em pesquisas ${ }^{14,15,20,22,28,29,31,33,34}$; a necessidade de se validarem outros tipos de evidências no campo da saúde ${ }^{21,29}$.

Os estudos do tipo "Editorial” mostram uma conceituação ampla e diferenciada da definição de MAC. Novamente os autores discutem as possibilidades e os limites da sua implementação, enfatizando a necessidade de maior investimento em pesquisas, assim como o ensino de MI nas escolas médicas. É consenso entre os autores que algumas modalidades de MAC são efetivas para certos tratamentos, além do fato de que o modelo filosófico de MI é perfeitamente aplicável no mundo real. Os autores reforçam a necessidade de a MI ter validação de estudos longitudinais clínicos, embora validem evidências relativas a mudança de estilo de vida, eficácia e custoefetividade ${ }^{11,13,27,32}$. 
O ensino de MI nas escolas médicas é apontado como um investimento necessário para a implementação do modelo de MI, contribuindo, durante a graduação ou pós-graduação, para o resgate de valores do núcleo da medicina ${ }^{17,40}$, além de acrescentar novas ferramentas ao trabalho, incluindo a comunicação, o trabalho em equipe e maior autonomia do paciente ${ }^{11,13}$. Os autores também destacam o crescente aumento dos usuários de MAC devido à insatisfação com o atual modelo de assistência à saúde ${ }^{9,17,27}$.

Quadro 1. Caracterização dos artigos analisados, segundo ano de publicação, local da realização, formação do primeiro autor e tipo de publicação.

\begin{tabular}{|c|c|c|c|c|c|}
\hline Referência & Ano & Local & $\begin{array}{c}\text { Formação do } \\
\text { primeiro } \\
\text { autor }\end{array}$ & Tipo de produção & $\begin{array}{l}\text { Total de } \\
\text { artigos } \\
\text { por ano }\end{array}$ \\
\hline $\operatorname{Rosch}^{8}$ & 1998 & USA & Médico & Revisão de literatura & \multirow[t]{2}{*}{2} \\
\hline Fontanarosa e Lundberg ${ }^{9}$ & 1998 & USA & Médico & Revisão de literatura & \\
\hline Chen e $\mathrm{Yu}^{10}$ & 1999 & China & Médico & Revisão de literatura & \multirow[t]{4}{*}{4} \\
\hline Foley $^{11}$ & 1999 & USA & Médico & Revisão de literatura & \\
\hline Dalen $^{12}$ & 1999 & USA & Médico & Revisão de literatura & \\
\hline Maizes e Caspi ${ }^{13}$ & 1999 & USA & Médica & Revisão de literatura & \\
\hline Ross et al..$^{14}$ & 2001 & USA & $\begin{array}{c}\text { Médico- } \\
\text { veterinário }\end{array}$ & Revisão de literatura & \multirow[t]{4}{*}{4} \\
\hline Hughes $^{15}$ & 2001 & USA & Médica & Revisão de literatura & \\
\hline Shang $^{16}$ & 2001 & USA & Médico & Revisão de literatura & \\
\hline Rees e Weil ${ }^{17}$ & 2001 & USA & Médico & Revisão de literatura & \\
\hline Hageness et al. ${ }^{18}$ & 2002 & USA & Enfermeira & Revisão de literatura & \multirow[t]{9}{*}{9} \\
\hline Ernst $^{19}$ & 2002 & Inglaterra & Médico & Revisão de literatura & \\
\hline Bell et al. ${ }^{20}$ & 2002 & USA & Médico & Revisão de literatura & \\
\hline Maizes et al. ${ }^{21}$ & 2002 & USA & Médica & Revisão de literatura & \\
\hline Maizes et al. ${ }^{22}$ & 2002 & USA & Médica & Revisão de literatura & \\
\hline Benor $^{23}$ & 2002 & USA & Médico & Revisão de literatura & \\
\hline Cohen e Eisenberg ${ }^{24}$ & 2002 & USA & Médico & Revisão de literatura & \\
\hline Marcus $^{25}$ & 2002 & USA & Médico & Revisão de literatura & \\
\hline Snyderman e Weil ${ }^{26}$ & 2002 & USA & Médico & Revisão de literatura & \\
\hline Easthope $^{27}$ & 2003 & Austrália & Sociólogo & Revisão de literatura & \multirow[t]{5}{*}{5} \\
\hline Cohen $^{28}$ & 2003 & USA & Médico & Revisão de literatura & \\
\hline Barrett et al. ${ }^{29}$ & 2003 & USA & Médico & Revisão de literatura & \\
\hline Caspi et al. ${ }^{30}$ & 2003 & USA & Médico & Editorial & \\
\hline Girman et al. ${ }^{31}$ & 2003 & USA & Médica & Editorial & \\
\hline Verhoef $^{32}$ & 2004 & Canadá & Médica & Editorial & \multirow[t]{7}{*}{7} \\
\hline Ernst $^{33}$ & 2004 & Reino Unido & Médico & Editorial & \\
\hline Boon et al. ${ }^{34}$ & 2004 & Canadá & Médico & Editorial & \\
\hline Hyman $^{35}$ & 2004 & USA & Médico & Editorial & \\
\hline Kligler et al. ${ }^{36}$ & 2004 & USA & Médico & Editorial & \\
\hline Mulkins e Verhoef ${ }^{37}$ & 2004 & Canadá & Médica & Editorial & \\
\hline Freeman et al. ${ }^{38}$ & 2004 & USA & Médica & Editorial & \\
\hline Milstein ${ }^{39}$ & 2005 & USA & Médico & Carta & \multirow[t]{5}{*}{5} \\
\hline Dalen $^{40}$ & 2005 & USA & Médico & Carta & \\
\hline Myers e Jacobsen ${ }^{41}$ & 2005 & USA & Médica & Carta & \\
\hline Hassed $^{42}$ & 2005 & Austrália & Médico & Ensaio clínico & \\
\hline Schiff et al. ${ }^{43}$ & 2005 & USA & Médico & Debate & \\
\hline
\end{tabular}


Quadro 2. Tipo de produção "Revisão da literatura".

\begin{tabular}{|c|c|}
\hline Referência & Definições de Medicina Integrativa \\
\hline Rosch $^{8}$ & $\begin{array}{l}\text { A meta da MI é promover uma boa saúde, e isso depende de um sistema de boa } \\
\text { comunicação. A MI implica a habilidade de extrair partículas de informações } \\
\text { que são aparentemente diferentes do assunto e sintetizá-las em algo que seja } \\
\text { significativo. A integração pertence à prática da medicina e significa restaurar } \\
\text { como um todo e renovar. }\end{array}$ \\
\hline Chen e $\mathrm{Yu}^{10}$ & $\begin{array}{l}\text { A medicina integrativa chinesa é a integração da medicina tradicional com a } \\
\text { medicina moderna. É um modelo que herda e desenvolve a medicina tradicional } \\
\text { por meio da ciência e da tecnologia médica moderna. }\end{array}$ \\
\hline Ross et al. ${ }^{14}$ & $\begin{array}{l}\text { A MI é a fronteira, é o futuro. É uma combinação do cuidado à saúde } \\
\text { convencional e não convencional. }\end{array}$ \\
\hline Hughes $^{15}$ & $\begin{array}{l}\text { Foca na saúde e na cura e não somente na doença e no tratamento. Vê o } \\
\text { paciente como uma pessoa completa: mente, corpo e espírito. Enfatiza o paci- } \\
\text { ente como provedor da relação e a prevenção de doenças. }\end{array}$ \\
\hline Hagenesset al. ${ }^{18}$ & $\begin{array}{l}\text { MI sugere que as terapias convencionais e as não convencionais sejam usadas em } \\
\text { conjunto para um maior potencial de cura do paciente. Ambas podem ser utili- } \\
\text { zadas como tratamento de primeira linha. }\end{array}$ \\
\hline Bell et al. ${ }^{20}$ & $\begin{array}{l}\text { É um sistema de cuidado primário, compreensivo, que enfatiza a saúde e a cura } \\
\text { da pessoa como um todo. Considera a saúde como uma propriedade emergente } \\
\text { da pessoa em um contexto ambiental. Utiliza recursos da boa prática médica, } \\
\text { sendo esses convencionais ou alternativos. Oferece maior possibilidade de con- } \\
\text { trole e cura do paciente. }\end{array}$ \\
\hline Maizes et al. ${ }^{21}$ & $\begin{array}{l}\text { MI é uma nova abordagem para medicina que honra a habilidade inata do corpo } \\
\text { se curar. Integra a MAC quando adequada para facilitar a cura. Os pacientes são } \\
\text { tratados como pessoas inteiras (corpo, mente, espírito). A comunicação e o } \\
\text { relacionamento são pontos centrais. A MI trabalha com uma definição ampla de } \\
\text { saúde, enfatiza a prevenção de doenças. }\end{array}$ \\
\hline Maizes et al. ${ }^{22}$ & $\begin{array}{l}\text { É a medicina orientada para a cura. Reenfatiza o relacionamento entre o pacien- } \\
\text { te e o médico e integra o melhor da MAC com a medicina convencional. MI é } \\
\text { definida muito mais amplamente do que MAC. }\end{array}$ \\
\hline Benor $^{23}$ & O cuidado integrativo combina as terapias convencionais com terapias MAC. \\
\hline Cohen e Eisenberg ${ }^{24}$ & $\begin{array}{l}\text { Cuidado complementar e integrativo é a integração clínica da biomedicina e } \\
\text { MAC. }\end{array}$ \\
\hline Snyderman e Weil ${ }^{26}$ & $\begin{array}{l}\text { MI é o novo termo para descrever um modelo de saúde que considera o } \\
\text { indivíduo e o desejo do consumidor. Sua missão é restaurar o foco da medicina } \\
\text { na saúde e não na doença. Enfatiza o paciente como centro da relação } \\
\text { terapêutica, a prevenção e os aspectos do estilo de vida. }\end{array}$ \\
\hline Cohen $^{28}$ & $\begin{array}{l}\text { A abordagem integrativa ou complementar consiste na adição das terapias con- } \\
\text { vencionais com as terapias alternativas. }\end{array}$ \\
\hline Barrett et al. ${ }^{29}$ & $\begin{array}{l}\text { MI resulta da forte incorporação de conceitos, valores e práticas das medicinas } \\
\text { convencional, complementar e alternativa. O processo de evolução da integra- } \\
\text { ção da MAC com a medicina convencional evoca novos moldes conceituais e } \\
\text { nova terminologia. Os termos complementar, alternativo e integrativo referem- } \\
\text { se a um grupo extremamente diverso de modalidades terapêuticas, os quais têm } \\
\text { pouco em comum com os outros e são diferentes da biomedicina. }\end{array}$ \\
\hline Caspi et al. ${ }^{30}$ & $\begin{array}{l}\text { O termo MI surgiu em resposta à necessidade de orientação da medicina mais } \\
\text { para a cura do que para a doença. É aberta a novos paradigmas, não rejeita a } \\
\text { medicina convencional e não aceita sem críticas a MAC. MI é um compreensivo } \\
\text { sistema de medicina, não somente em técnicas, mas em fundação conceitual, } \\
\text { diferente da perspectiva da ciência clássica na saúde, como ausência de doença } \\
\text { física. Coloca o paciente no centro da relação terapêutica, dá importância ao } \\
\text { ponto de vista do paciente. }\end{array}$ \\
\hline
\end{tabular}




\begin{tabular}{|c|c|c|}
\hline \multirow[t]{10}{*}{ 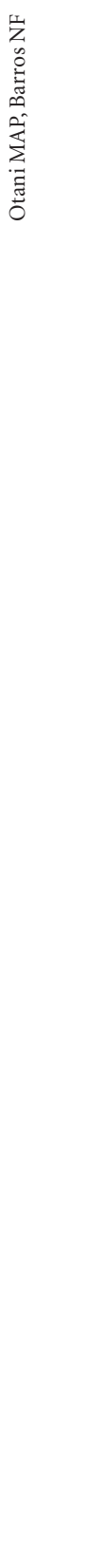 } & Quadro 2. continua & \\
\hline & Referência & Definições de Medicina Integrativa \\
\hline & Girman et al. ${ }^{31}$ & $\begin{array}{l}\text { Uma abordagem integrativa para o cuidado da saúde usa a combinação das mais } \\
\text { apropriadas terapias convencionais e não convencionais para tratar determinada } \\
\text { situação. Depende dos recursos internos do paciente para superar problemas. O } \\
\text { profissional auxilia o paciente na percepção e no uso desse sistema de tratamento. }\end{array}$ \\
\hline & Ernst $^{33}$ & $\begin{array}{l}\text { A criação do termo MI insinua que a maioria dos médicos é incapaz de abordar a } \\
\text { pessoa como um todo e vê seus pacientes por partes. A medicina científica e o } \\
\text { holismo não são incompatíveis, podem se complementar em harmonia. O termo } \\
\text { MI é supérfluo ao duplicar conceitos que estão no coração da medicina. }\end{array}$ \\
\hline & Boon et al. ${ }^{34}$ & $\begin{array}{l}\text { Define cuidado integrativo à saúde com base em quatro temas: Filosofia e } \\
\text { valores: trata a pessoa como um todo, avalia suas propriedades inatas, promove } \\
\text { saúde e previne doenças; Estrutura: relação não hierárquica e interdisciplinar da } \\
\text { medicina convencional com a MAC para o cuidado centrado no paciente; } \\
\text { Processo: abordagem em equipe com a construção de um consenso, respeito } \\
\text { mútuo e uma visão ampliada do cuidado à saúde; Resultados: cuidado mais } \\
\text { efetivo e custo efetivo. }\end{array}$ \\
\hline & Kligler et al. ${ }^{36}$ & $\begin{array}{l}\text { É uma abordagem da prática da medicina que faz uso da melhor evidência dispo- } \\
\text { nível, levando em consideração a pessoa por inteiro (mente, corpo e espírito), } \\
\text { incluindo aspectos do estilo de vida. Enfatiza o relacionamento terapêutico e faz } \\
\text { uso tanto da abordagem convencional quanto da alternativa e complementar. }\end{array}$ \\
\hline & Freeman et al. ${ }^{38}$ & $\begin{array}{l}\text { Uma abordagem de MI baseada em evidências une opções de tratamento com } \\
\text { eficácia comprovada e o desejo público pelos tratamentos da medicina alternativa } \\
\text { e complementar. }\end{array}$ \\
\hline & Milstein $^{39}$ & $\begin{array}{l}\text { Modelo de cuidado integrativo: propõe um acompanhamento contínuo integran- } \\
\text { do elementos do modelo biopsicossocial com compaixão, incorporando a cura e o } \\
\text { tratamento de doenças e a experiência humana do indivíduo. }\end{array}$ \\
\hline & Hassed $^{42}$ & $\begin{array}{l}\text { A MI envolve uma variedade de opções de tratamento, incluindo cuidado conven- } \\
\text { cional, controle do estilo de vida, abordagens psicossociais e a medicina comple- } \\
\text { mentar baseada em evidências. Uma abordagem holística que adiciona as terapias } \\
\text { complementares à farmacologia convencional; oferece maior potencial de con- } \\
\text { trole dos sintomas e autocontrole dos pacientes. }\end{array}$ \\
\hline & Schiff et al. ${ }^{43}$ & $\begin{array}{l}\text { É a medicina orientada para a cura que considera a pessoa como um todo (corpo, } \\
\text { mente e espírito), incluindo os aspectos do estilo de vida. Enfatiza o relaciona- } \\
\text { mento terapêutico e faz uso apropriado tanto das terapias convencionais quanto } \\
\text { das terapias alternativas. }\end{array}$ \\
\hline
\end{tabular}

Nos estudos do tipo "Carta", "Debate" e "Ensaio clínico", não há consenso entre os autores em relação ao conceito de MI. Alguns conceituam amplamente $\mathrm{MI}$, ressaltando a necessidade de valorização das evidências atuais da $\mathrm{MAC}^{12,16}$ e a participação do paciente no tratamento ${ }^{12,37}$. Os outros autores questionam a criação de novos termos, assim como a possibilidade de aplicação na prática ${ }^{12,19,25}$. Dentre as sugestões e os comentários tecidos por esses autores, destacamse: a importância do ensino de MAC nas escolas médicas ${ }^{12,25}$ e a necessidade de maior investimento em pesquisas sobre MI, com a utilização de metodologias que favoreçam a avaliação da eficácia e custo-efetividade ${ }^{16,19,25}$.

\section{Discussão}

O objetivo deste trabalho é fomentar o debate sobre a Medicina Integrativa, apresentando uma síntese dos artigos publicados sobre o tema nos últimos anos. Os limites deste trabalho são claros na medida em que ele desenvolveu análise de dados secundários e privilegiou uma única base de dados, embora o Medline/Pubmed seja o mais reconhecido e validado banco de referências bibliográficas do campo da saúde.

Um dos aspectos mais significativos na literatura analisada é o destaque dado ao aumento do número de consumidores e fornecedores de MAC, associado à insatisfação com a organização do 
Quadro 3. Tipo de produção "Editorial”.

\begin{tabular}{|c|c|}
\hline Referência & Definições de Medicina Integrativa \\
\hline $\begin{array}{l}\text { Fontanarosa e } \\
\text { Lundberg }^{9}\end{array}$ & $\begin{array}{l}\text { As numerosas práticas que levam o termo de medicina complementar, alterna- } \\
\text { tiva, não convencional ou integrativa englobam várias modalidades terapêuticas } \\
\text { e filosofias que geralmente são consideradas fora da medicina convencional. }\end{array}$ \\
\hline Foley $^{11}$ & $\begin{array}{l}\text { Saúde integrativa é a combinação das disciplinas médicas convencionais com as } \\
\text { terapias complementares e alternativas, formando um continuum das ferramen- } \\
\text { tas de administração da saúde e de doenças dentro de uma estrutura de padrões } \\
\text { profissionais aceitos. Permite aos médicos considerarem as condições genéticas, } \\
\text { ambientais, nutricionais, psicossociais, estressantes e crenças de cada paciente. } \\
\text { Enfatiza o ser humano. }\end{array}$ \\
\hline Maizes e Caspi ${ }^{13}$ & $\begin{array}{l}\text { MI valoriza a capacidade inata do corpo para a cura. Requer mudança de paradig- } \\
\text { ma da doença à saúde. Um de seus princípios é o relacionamento terapêutico que } \\
\text { facilita o processo de cura e focaliza as necessidades individuais do paciente. Enfa- } \\
\text { tiza a participação do paciente no tratamento e a prevenção de doenças. Mantém } \\
\text { o paciente no foco do tratamento e multiplica as estratégias disponiveis. }\end{array}$ \\
\hline Rees e Weil ${ }^{17}$ & $\begin{array}{l}\text { A MI tem seu foco na saúde e na cura mais do que na doença e no tratamento. } \\
\text { Enxerga o paciente como um todo (corpo, mente e espírito) e considera fatores } \\
\text { do estilo de vida, incluindo dieta, exercício, qualidade do descanso e sono e } \\
\text { natureza dos relacionamentos. }\end{array}$ \\
\hline Easthope $^{27}$ & $\begin{array}{l}\text { Houve evolução dos conceitos; no início era chamada de medicina alternativa, } \\
\text { depois complementar, e hoje faz parte da MI. Associa a validação da MI ao } \\
\text { aumento da utilização da medicina baseada em evidências, o que ajuda a de- } \\
\text { monstrar cientificamente que ela funciona. }\end{array}$ \\
\hline Verhoef ${ }^{32}$ & $\begin{array}{l}\text { MI é um sistema inteiro que inclui o paciente como provedor da relação, multi- } \\
\text { plicando os tratamentos convencionais com a MAC e o contexto filosófico de } \\
\text { cuidar como uma intervenção. Os resultados sistêmicos englobam mudanças } \\
\text { simultâneas e interativas da pessoa como um todo. }\end{array}$ \\
\hline Hyman $^{35}$ & $\begin{array}{l}\text { A MI tem como um princípio a inter-relação das forças biológicas, sociais, } \\
\text { psicológicas e espirituais levando ao desequilíbrio ou a doenças. A MI vem para } \\
\text { transformar o sistema de cuidado à doença em sistema de cuidado à saúde. }\end{array}$ \\
\hline Dalen $^{40}$ & $\begin{array}{l}\text { A MI valoriza a relação mente-corpo e enfatiza a prevenção. O médico deve ser } \\
\text { capaz de aconselhar seus pacientes sobre nutrição, vitaminas e suplementos, } \\
\text { assim como as indicações e possíveis benefícios da MAC. }\end{array}$ \\
\hline Myers e Jacobsen ${ }^{41}$ & $\begin{array}{l}\text { MI é a integração de terapias alternativas e complementares baseada em evidên- } \\
\text { cias com o cuidado convencional. Suas metas são: enfatizar a promoção da } \\
\text { saúde, prevenir doenças, melhorar os resultados dos pacientes, gerenciar sinto- } \\
\text { mas e melhorar a qualidade de vida. }\end{array}$ \\
\hline
\end{tabular}

atual sistema de saúde, a fragmentação do cuidado e o desejo de tratamentos mais suaves e com menos riscos de efeitos adversos. Esse movimento social data de algumas décadas, mas a produção de conceitos e definições referentes a ele ainda estão em processo, sendo a proposta da Medicina Integrativa mais uma tentativa de abranger vários aspectos, como a integração da medicina alternativa e complementar com a medicina convencional; a combinação de sistemas antigos de cura com a biomedicina moderna; a valorização do relacionamento médico-paciente e da comu- nicação; a consideração da pessoa de forma integral; a utilização de evidências; e o enfoque na saúde, na cura e na prevenção de doenças.

Todavia, esse conjunto de fatores tem levado a uma disputa conceitual no campo da saúde, pois alguns autores identificam a Medicina Integrativa como a combinação da Medicina Convencional e da Medicina Alternativa e Complementar, enquanto outros identificam a Medicina Integrativa como um novo paradigma mais abrangente, com alcance além da simples combinação de diferentes modalidades de tratamento. 


\begin{tabular}{|c|c|c|}
\hline \multirow[t]{7}{*}{ 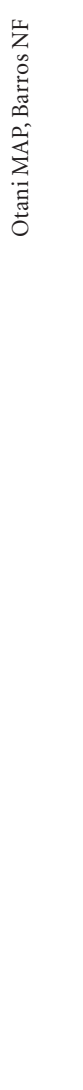 } & Quadro 4. Tipo de pr & ão "Carta", "Ensaio clínico" e "Debate". \\
\hline & Referência & Definições de Medicina Integrativa \\
\hline & Shang ${ }^{16}$ & $\begin{array}{l}\text { A abordagem da MI pode unificar as diferentes teorias dentro da medicina não } \\
\text { convencional e ser a ponte entre a medicina convencional e não convencional } \\
\text { com benefícios mútuos. }\end{array}$ \\
\hline & Ernst $^{19}$ & $\begin{array}{l}\text { MI não é nova, é idêntica à velha medicina holística, existindo durante toda a } \\
\text { história da medicina. Questiona a viabilidade do modelo de MI ante a realidade } \\
\text { econômica da saúde e a necessidade de maiores evidências. Concorda que os } \\
\text { princípios centrais da MI não são novos. Eles podem e devem ser aplicados em } \\
\text { todos os campos da medicina. Considera relevante a complexidade do conceito } \\
\text { e implicações para a prática do cuidado à saúde e os resultados das pesquisas. }\end{array}$ \\
\hline & Marcus $^{25}$ & $\begin{array}{l}\text { Não há diferença fundamental entre a medicina convencional e a integrativa, } \\
\text { visto que a medicina convencional também se preocupa com o estilo de vida } \\
\text { saudável para a prevenção e o controle das doenças. }\end{array}$ \\
\hline & Mulkins e Verhoef $^{37}$ & $\begin{array}{l}\text { O cuidado de saúde integrativa tem como objetivo recuperar o equilíbrio físico, } \\
\text { emocional, estrutural, energético e espiritual baseado na habilidade inata do } \\
\text { corpo para se curar. A MI muda o paradigma da doença para a saúde, mantendo } \\
\text { o paciente no foco central do cuidado, e multiplica o número de estratégias } \\
\text { disponíveis ao paciente. }\end{array}$ \\
\hline & Dalen $^{12}$ & $\begin{array}{l}\text { A MI combina a MAC com a medicina convencional. É o futuro, e as instituições } \\
\text { não sobreviverão sem ela. A MI pode significar um retorno a passado, } \\
\text { interrompendo o extraordinário progresso da medicina baseada na ciência. }\end{array}$ \\
\hline
\end{tabular}

\section{Medicina Integrativa é semelhante à Medicina Alternativa e Complementar}

Ao discutir o que é alternativo e para quem é alternativo, autores como Cohen e Eisenberg ${ }^{24}$ refletem sobre o fato de que o alternativo para uns pode ser principal para outros e, por isso, propõem que o foco operacional das definições de práticas não convencionais centre-se, também, no indivíduo e não apenas no coletivo.

Para a maioria dos autores analisados, no entanto, a definição de Medicina Integrativa está relacionada com a integração da medicina convencional com a medicina não convencional, com o objetivo de oferecer melhor cuidado ao paciente, dando a estes a oportunidade de optar pela forma mais adequada para seu tratamento. Com a adoção deste ponto de vista, eles questionam a criação de novos nomes e conceitos, uma vez que consideram o holismo e a medicina científica como práticas não incompatíveis, sendo possível a sua integração, sobretudo a partir de evidências científicas.

Ainda de acordo com esses autores, a Medicina Integrativa fundamenta-se na abordagem holística, isto é, na compreensão do homem como um todo indivisível, impossível de ser se- parado em corpo físico, mente e espírito, com ênfase na cura, no relacionamento interpessoal e no contexto de vida. Essa visão do ser humano funciona como antítese do modelo biomédico mecanicista, que privilegia as partes da máquina humana e os processos bioquímicos que a fazem funcionar. Assim, a abordagem Integrativa estimula os profissionais a considerarem em cada paciente os fatores genéticos, ambientais, nutricionais, psicossociais, estressantes e culturais associados a crenças, valores e símbolos. Estimula, por fim, a ênfase na prevenção de doenças e promoção de saúde, afirmando que se trata de práticas existentes desde a origem da medicina moderna, porém negligenciadas.

\section{Medicina Integrativa é diferente de Medicina Alternativa e Complementar}

Para outro grupo de autores, o conceito de Medicina Integrativa está associado a uma mudança de paradigma. Assim, para exercê-la é necessário modificar o pensamento em relação à saúde, ou seja, mudar o conceito, as formas de intervenção em relação ao processo saúde-doença e o modelo de atenção à saúde. 
O fato de dimensionarem a Medicina Integrativa como paradigma traz-lhes um conjunto de questões diferentes das anteriores, sobretudo associadas às dificuldades para sua implantação. Muitas são as barreiras elencadas, como o tempo das consultas; o número de profissionais e o fluxo de pacientes; a falta de evidências científicas; as disputas organizacionais; a mudança na abordagem pessoal; o maior tempo para se observarem os efeitos das técnicas não convencionais; e o pequeno investimento em pesquisas.

Alguns autores deste grupo observam que houve aumento do número de pesquisas sobre o tema, sobretudo procurando construir evidências para o uso. Entretanto, refletem sobre os limites das pesquisas que adotam a metodologia quantitativa da ciência normal, admitindo que a eficiência e a eficácia das intervenções podem ser observadas, também, a partir das evidências produzidas de forma qualitativa e centradas no paciente. Por isso, esses autores reafirmam a necessidade de mudanças na formação dos profissionais da saúde, que deve promover novos elementos na perspectiva do cuidado, por meio do ensino e da prática de MAC nos currículos. Para alguns autores, a informação sobre a MAC nos cursos de graduação já seria suficiente, desde que garantisse conhecimento para orientação aos pacientes, ao passo que a formação para execução de tais terapias deve ser parte do processo de profissionalização.

Diante disso, há um consenso neste grupo de que a integração das Medicinas Convencional, Alternativa e Complementar deva acontecer dentro dos padrões ético-legais atuais, observando o que cada uma tem de melhor, para que políticas públicas sejam criadas e investimentos garantidos.

\section{Considerações finais}

Da análise dos artigos, conclui-se que a maior justificativa para se investigarem as práticas não convencionais, no campo da saúde, está no número de pacientes e profissionais que se interessam por elas, além dos movimentos sociais de legitimação e legalização dessas práticas. No Brasil, ainda não se realizou um estudo nacional so- bre o uso das práticas não convencionais, embora investigações em serviços de saúde com pacientes em tratamento de câncer e diabetes tenham mostrado o uso de medicina tradicional/MAC por mais de $60 \%$ dos entrevistados. No âmbito do SUS, uma iniciativa fundamental foi a criação da Política Nacional de Práticas Integrativas e Complementares (PNPIC), em 2006, buscando ampliar a oferta e o acesso à prática da acupuntura, da homeopatia, da fitoterapia e do termalismo ${ }^{44}$.

Conclui-se ainda que há uma divisão entre os autores em relação ao entendimento do que é a Medicina Integrativa. No entanto, entre as duas perspectivas acreditamos que, apesar da maior dificuldade e do maior tempo, prevalecerá a noção da Medicina Integrativa como diferente de Medicina Alternativa e Complementar, portanto como novo paradigma no campo da saúde. Sobretudo porque ela tem forte identidade com outros importantes debates, como integralidade do cuidado, humanização das relações, construção de evidências científicas e mudanças na educação em saúde.

Com base na perspectiva integrativa, acreditamos que a curto prazo o modelo de atenção à saúde poderá ter custo mais elevado, em razão das mudanças na organização do sistema de saúde e nas percepções dos profissionais sobre o processo saúde-doença. Porém, a médio e longo prazos, a criação de serviços integrados levará à diminuição de gastos, devido ao cuidado integral, prevenção de doenças e promoção da saúde com que opera.

\section{Colaboradores}

MAP Otani e NF Barros participaram igualmente de todas as etapas da elaboração do artigo. 


\section{Referências}

1. Luz MT, Rosenbaum P, Barros NF. Medicina Integrativa, política pública de saúde conveniente. Jornal da Unicamp 2006; 27 ago. p. 2.

2. Giddens A. Sociologia. $4^{\text {a }}$ ed. Porto Alegre: Artmed; 2005.

3. Barros NF. A construção de novos paradigmas na medicina: a medicina alternativa e a medicina complementar. In: Canesqui AM, organizadora. Ciências sociais e saúde para o ensino médico. São Paulo: Hucitec; 2000. p. 201-213.

4. Teixeira MZ, Lin CA, Martins MA. O ensino das práticas não-convencionais em saúde nas faculdades de medicina: panorama mundial e perspectivas brasileiras. Rev Bras Educ Med 2004; 28(1):51-60.

5. National Center for Complementary and Alternative Medicine. What is CAM? [acessado 2007 maio 23]. Disponível em: http://nccam.nih.gov/health/ whatiscam/

6. Ferreira ABH. Novo dicionário da língua portuguesa $2^{a}$ ed. Rio de Janeiro: Nova Fronteira; 1986. Verbete integração; p. 954.

7. Vieira S, Hossne WS. Metodologia científica para a área da saúde. Rio de Janeiro: Campus; 2001.

8. Rosch PJ. Integrative thinking: the essence of good medical education and practice. Integr Physiol Behav Sci 1998; 33(2):141-150.

9. Fontanarosa PB, Lundberg GD. Complementary, alternative, unconventional and integrative medicine: call for papers for the annual coordinated theme issues of the AMA Journals. Arch Gen Psychiatry 1998; 55(1):82-83.

10. Chen K, Yu B. Certain progress of clinical research on Chinese integrative medicine. Chin Med J (Engl) 1999; 112(10):934-937.

11. Foley C. Patient demand for integrative medicine. Minn Med 1999; 82(5):50-51.

12. Dalen JE. Is integrative medicine the medicine of the future?: a debate between Arnold S. Relman and Andrew Weil. Arch Intern Med 1999; 159(18):2122-2126.

13. Maizes V, Caspi O. The principles and challenges of integrative medicine: more than a combination of traditional and alternative therapies. West J Med 1999; 171(3):148-149.

14. Ross C, Haussler KK, Kenney JD, Marks D, Bertone JJ, Henneman K, May KJ. Frontier medicine: the future and integrative medicine. Vet Clin North $\mathrm{Am}$ Equine Pract 2001; 17(2):351-377.

15. Hughes EF. Overview of complementary, alternative, and integrative medicine. Clin Obstet Gynecol 2001; 44(4):774-779.

16. Shang C. The future of integrative medicine. Arch Intern Med 2001; 161(4):613-614.
17. Rees L, Weil A. Integrated medicine: imbues orthodox medicine with the values of complementary medicine. BJM 2001; 322(7279):119-120.

18. Hageness SM, Kreitzer MJ, Kinney ME. Complementary, integrative and holistic care in emergency nursing. Nurs Clin North Am 2002; 37(1):123-133.

19. Ernst E. Integrative medicine: not a carte blanche for untested nonsense [letter]. Arch Intern Med 2002; 162(15):1781

20. Bell IR, Caspi O, Schwartz GE, Grant KL, Gaudet TW, Rychener D, Maizes V, Weil A. Integrative medicine and systemic outcomes research: issues in the emergence of a new model for primary health care. Arch Intern Med 2002; 162(2):133-140.

21. Maizes V, Koffler K, Fleishman S. Revisiting the health history: an integrative medicine approach. Adv Mind Body Med 2002; 18(2):31-34.

22. Maizes V, Schneider C, Bell IR, Weil A. Integrative medical education: development and implementation of a comprehensive curriculum at the University of Arizona. Acad Med 2002; 77(9):851-860.

23. Benor DJ. Energy medicine for the internist. Med Clin North Am 2002; 86(1):105-125.

24. Cohen MH, Eisenberg DM. Potential physician malpractice liability associated with complementary and integrative medical therapies. Ann Intern Med 2002; 136(8):596-603.

25. Marcus DM. Integrative medicine is a trojan horse. Arch Intern Med 2002; 162(20):2381-2383.

26. Snyderman R, Weil AT. Integrative medicine: bringing medicine back to its roots. Arch Intern Med 2002; 162(4):395-397.

27. Easthope G. Alternative, complementary or integrative? Complement Ther Med 2003; 11(1):2-3.

28. Cohen MH. Complementary and integrative medical therapies, the FDA, and the NIH: definitions and regulation. Dermatol Ther 2003; 16(2):77-84.

29. Barrett B, Marchand L, Scheder J, Plane MB, Maberry R, Appelbaum D, Rakel D, Rabago D. Themes of holism, empowerment, access, and legitimacy define complementary, alternative and integrative medicine in relation to conventional biomedicine. J Altern Complement Med 2003; 9(6):937-947.

30. Caspi O, Sechrest L, Pitluk HC, Marshall CL, Bell IR, Nichter M. On the definition of complementary, alternative and integrative medicine: societal mega-stereotypes vs. the patients' perspectives. Altern Ther Health Med 2003; 9(6):58-62.

31. Girman A, Lee R, Kligler B. An integrative medicine approach to premenstrual syndrome. $A m \mathrm{~J}$ Obstet Gynecol 2003; 188(Suppl. 5):S56-S65.

32. Verhoef MJ. Evaluating integrative medicine: science and art: commentary on "a descriptive analysis of an integrative medicine clinic". J Altern Complement Med 2004; 10(4):595-596. 
33. Ernst E. Disentangling integrative medicine. Mayo Clin Proc 2004; 79(4):565-566.

34. Boon H, Verhoef M, O'Hara D, Findlay B, Majid N. Integrative healthcare: arriving at a working definition. Altern Ther Health Med 2004; 10(5):48-56.

35. Hyman MA. Integrative health and medicine: an opportunity for leadership and collaboration. Altern Ther Health Med 2004; 10(3):10-11.

36. Kligler B, Maizes V, Schachter S, Park CM, Gaudet T, Benn R, Lee R, Remen RN. Core competencies in integrative medicine for medical school curricula: a proposal. Acad Med 2004; 79(6):521-531.

37. Mulkins AL, Verhoef MJ. Supporting the transformative process: experiences of cancer patients receiving integrative care. Integr Cancer Ther 2004; 3(3):230-237.

38. Freeman MP, Helgason C, Hill RA. Selected integrative medicine treatments for depression: considerations for women. Journal of the American Medical Women's Association 2004; 59(3):216-224.

39. Milstein J. A paradigm of the integrative care: healing with curing throughout life, "being with" and “doing to". Journal of Perinatology 2005; 25:563-568.

40. Dalen JE. How can a conventionally trained physician support Integrative Medicine? Alternative Therapies 2005; 11(1):10-11.

41. Myers C, Jacobsen P. Integrative medicine and oncology: emerging evidence. Cancer control: Journal of the Moffitt Cancer Center 2005; 12(3):146-147.

42. Hassed C. An integrative approach to asthma. Australian Family Physician 2005; 34(7):573-576.

43. Schiff E, Kim YH, Maizes V. Vegetative states: an integrative approach. Alternative Therapies 2005; 11(1):26-33.

44. Brasil. Ministério da Saúde. Secretaria de Atenção a Saúde. Departamento de Atenção Básica. Política Nacional de Práticas Integrativas e Complementares no SUS - PNPIC-SUS. Brasília: Ministério da Saúde; 2006.

Artigo apresentado em 20/05/2008

Aprovado em 06/10/2008

Versão final apresentada em 07/11/2008 